\title{
MicroRNA-205-5p suppresses the invasiveness of oral squamous cell carcinoma by inhibiting TIMP-2 expression
}

\author{
HIROKI NAGAI ${ }^{1}$, SHOGO HASEGAWA $^{1,6}$, FUMIHIKO UCHIDA $^{2}$, TAKEHITO TERABE ${ }^{1}$, \\ NAOMI ISHIBASHI KANNO ${ }^{2}$, KOROKU KATO ${ }^{3}$, KENJI YAMAGATA ${ }^{2}$, SATOSHI SAKAI ${ }^{4}$, \\ SHUICHI KAWASHIRI $^{3}$, HIROSHI SATO ${ }^{5}$, TORU YANAGAWA ${ }^{2}$ and HIROKI BUKAWA ${ }^{2}$ \\ ${ }^{1}$ Department of Oral and Maxillofacial Surgery, Graduate School of Comprehensive Human Sciences, University of Tsukuba; \\ ${ }^{2}$ Department of Oral and Maxillofacial Surgery, Faculty of Medicine, University of Tsukuba, Tsukuba, \\ Ibaraki 305-8571; ${ }^{3}$ Department of Oral and Maxillofacial Surgery, Faculty of Medicine, Institute of Medical, \\ Pharmaceutical and Health Sciences, Kanazawa University, Kanazawa, Ishikawa 920-0934; \\ ${ }^{4}$ Cardiovascular Division, Faculty of Medicine, University of Tsukuba, Tsukuba, Ibaraki 305-8571; \\ ${ }^{5}$ Department of Molecular Virology and Oncology, Cancer Research Institute, \\ Kanazawa University, Kanazawa, Ishikawa 920-1192, Japan
}

Received August 17, 2017; Accepted December 7, 2017

DOI: $10.3892 /$ ijo. 2018.4260

\begin{abstract}
MicroRNAs (miRNAs or miRs) play important roles in carcinogenesis. The miRNA, miR-205-5p, has been reported to suppress the growth of various types of tumor; however, its functional contribution to oral squamous cell carcinoma (OSCC) is not yet clear. Thus, this study was conducted to determine the miRNA expression signatures in OSCC and to investigate the functional role of miR-205-5p in OSCC cells. We measured miR-205-5p expression by RT-qPCR, and examined the function of miR-205-5p by transfecting a miR-205-5p mimic or inhibitor into OSCC cells and measuring cell proliferation, migration and invasiveness. Genes targeted by miR-205-5p were identified using the TargetScan database and verified by western blot analysis, luciferase reporter assay and ELISA. We found that miR-205-5p was significantly downregulated in OSCC cell lines and tissue specimens. Following transfection of miR-205-5p mimic or inhibitor into the cancer cell lines, miR-205-5p overexpression significantly suppressed cancer cell migration and invasion. We further demonstrated that miR-205-5p directly targeted and regulated the tissue inhibitor of metalloproteinases-2 (TIMP-2) gene. The silencing of TIMP-2 suppressed cancer cell invasion and the activation of pro-matrix metalloproteinase-2 (pro-MMP-2). These results suggest that TIMP-2 promotes tumor progression, and that miR-205-5p directly regulates TIMP-2, thereby suppressing
\end{abstract}

Correspondence to: Dr Shogo Hasegawa, ${ }^{6}$ Present address: Department of Oral and Maxillofacial Surgery, Toyokawa City Hospital, Toyokawa, 23 Noji, Yawatacho, Aichi 442-8561, Japan

E-mail:shogo_h@icloud.com

Key words: invasion, microRNA-205-5p, matrix metalloproteinase-2 activation, oral squamous cell carcinoma, tissue inhibitor of metalloproteinases-2
pro-MMP-2 activation and inhibiting OSCC cell invasiveness. Our data describing the pathways regulated by miR-205-5p provide new insight into the mechanisms responsible for OSCC development and metastasis.

\section{Introduction}

Oral squamous cell carcinoma (OSCC) represents $>95 \%$ of cancers of the oral cavity (1). In developing countries, OSCC is the 6th most common type of cancer. OSCC has an overall 5 -year survival rate of $<50 \%$ (1), and this poor prognosis is due mainly to a high likelihood of metastasis to lymph nodes in the neck (2-5). Despite recent advances in treatment modalities, such as surgery, radiotherapy and chemotherapy, the survival rates for patients with OSCC have not markedly improved (6). Thus, metastasis to the lymph nodes in the neck is a major prognostic factor in OSCC.

Tumor invasion and metastasis require tumor cells to resolve and move through the extracellular matrix (ECM). Matrix metalloproteinases (MMPs) and tissue inhibitors of metalloproteinases (TIMPs) are important for the degradation of the ECM during these processes (7). MMP-2 is of particular importance in tumor-cell invasion as it can deconstruct collagen type IV, the major constituent of basement membranes (8). The proteolytic activation of MMP-2 is controlled by TIMP-2, which is necessary for the activation of pro-MMP-2 $(9,10)$. TIMP-2 forms a complex with membrane type-I metalloproteinase 1 (MT1-MMP) on cell membranes, and the TIMP-2/MT1-MMP complex activates pro-MMP-2. Thus, an imbalance in MMP-2 and TIMP-2 may disrupt the balance of ECM turnover and can thus lead to the uncontrolled degradation of the ECM, including basement membranes, and is the most likely cause of tumor invasion and metastasis (7). Thus, it may be possible to control pro-MMP-2 activation, and therefore tumor invasion and metastasis, by regulating TIMP-2. 
MicroRNAs (miRNAs or miRs), which are short, non-coding RNAs of 20-22 nucleotides in length, that regulate gene expression at the post-transcriptional level by interacting with the 3'-untranslated regions (3'-UTRs) of a target gene (11). miRNAs are involved in a wide range of biological functions, and can function as oncogenes or tumor suppressors, according to the functions of their target genes. To date, several miRNAs have been implicated in the progression of OSCC, including miR-26a/b, miR-23b/27b, miR-155, miR-221 and miR-205 (12-16). Recent studies have shown that miR-205-5p is downregulated in various cancer cells, including breast cancer, prostate cancer and oral cancer cells (17-19). In addition, the transient overexpression of miR-205-5p in cancer cells has been reported to suppress tumor progression by inhibiting tumor-associated genes and upregulating tumor suppressor genes. However, the signaling pathway and antitumorigenic functions of miR-205-5p in various cancer cells are unclear.

Therefore, the present study was conducted to determine the function of miR-205-5p in OSCC and the molecular mechanisms through which miR-205-5p suppresses OSCC tumor progression.

\section{Materials and methods}

OSCC clinical specimens and cell lines. OSCC cell lines (HSC3 and SAS) obtained from the Japanese Collection of Research Bioresources (Osaka, Japan) were cultured as previously described (14). We authenticated HSC3 and SAS through short tandem repeat (STR) profiling (PowerPlex 16 STR system) in 2017 and ensured that no culture contamination had occurred. We obtained 71 primary OSCC tissue, and 28 metastatic lymph node tissue samples from 71 previously untreated subjects with OSCC who visited the University of Tsukuba Hospital (Tsukuba, Japan) between February 2008 and November 2010, as well as tissues samples from 6 subjects who did not have cancer. Primary and metastatic specimens were collected at the time of biopsy and neck dissection, respectively. The samples were prepared for formalin-fixed paraffin-embedded (FFPE) histology using standard procedures. OSCC was diagnosed and classified based on the tumor-node metastasis (TNM) system of the Union for International Cancer Control (UICC). All OSCC cases were diagnosed clinically and confirmed histologically by pathologists. The clinical characteristics of the patients with OSCC are shown in Table I.

This study was reviewed and approved by the Ethics Committee University of Tsukuba Hospital (approval no. 215). All patients provided informed written consent prior to enrollment.

TaqMan-based reverse transcription-quantitative PCR (RT-qPCR) assays of miRNA expression. Total RNA was extracted using the miRNeasy Mini kit (Qiagen, Venlo, The Netherlands) for cell lines and the miR Neasy FFPE kit (Qiagen) for FFPE tissues. Reverse transcription was performed using the TaqMan MicroRNA Reverse Transcription kit (Applied Biosystems, Foster City, CA, USA). miR-205 expression was measured using the TaqMan MicroRNA assay system (Applied Biosystems) according to the manufacturer's instructions. PCR reactions were performed using the CFX384 Real-Time system (Bio-Rad Laboratories, Pleasanton, CA, USA). The small RNA, RNU6B, was used as an internal control. Relative expression was calculated using the comparative threshold $(\mathrm{Ct})$ method (20).

TaqMan-based RT-qPCR assay of mRNA expression. Total RNA was extracted using the RNeasy Mini kit (Qiagen) and reverse-transcribed using the PrimeScript RT Reagent kit (Takara, Shiga, Japan). PCR reactions were conducted using the CFX384 Real-Time system (Bio-Rad Laboratories). Relative mRNA expression was normalized against GAPDH. Relative expression was calculated by the comparative threshold (Ct) method $(20,21)$. The following primers were used for RT-qPCR: TIMP-2 forward, 5'-GCCCCCGCCCGC CCAGCCCCCC-3' and reverse, 5'-GCAACAATATCCACTT TACCAGAGTTAA-3'; and GAPDH forward, 5'-CAACGG ATTTGGTCGTATTGG-3' and reverse, 5'-GCAACAATATC CACTTTACCAGAGTTAA-3'.

Transfection with miR-205-5p mimic or inhibitor or with SiRNA. The cells were cultured to $70-80 \%$ confluence in 12-well plates and transfected with $50 \mathrm{nM}$ miR-205-5p mimic or inhibitor (hsa-miR-205 mirVana ${ }^{\mathrm{TM}}$ miRNA Mimic, \#4464066; mirVana $^{\mathrm{TM}}$ miRNA Inhibitor, \#4464084), or with $50 \mathrm{nM}$ scramble negative control $\left(\operatorname{mirVana}^{\mathrm{TM}}\right.$ miRNA Mimic Negative Control, \#4464058; mirVana $^{\mathrm{TM}}$ miRNA Inhibitor Negative Control, \#4464076) from Ambion (Austin, TX, USA). To knock down TIMP-2, the cells were transfected with 25 nM TIMP-2 siRNA (Silencer ${ }^{\circledR}$ Select siRNA, \#4390824) or control siRNA (Silencer ${ }^{\circledR}$ Negative control siRNA, \#AM4611). Lipofectamine RNAiMAX transfection reagent (Invitrogen, Carlsbad, CA, USA) was used according to the manufacturer's instructions. The cells were incubated at $37^{\circ} \mathrm{C}$ for $24 \mathrm{~h}$ after transfection, the medium was exchanged for FBS-free medium, and the cells were incubated for a further $48 \mathrm{~h}$. Both the transfected cells and the culture media were used for assays and analyses, as described below.

Cell proliferation, migration and invasion assays. The cells were cultured as previously described (14). Cell proliferation assay was performed as described in a previous study (22). The cells were transfected with $50 \mathrm{nM}$ miR-205-5p mimic or inhibitor by reverse transfection and plated in 96-well plates at $3 \times 10^{3}$ cells/well. After $72 \mathrm{~h}$, cell proliferation was determined by MTT assay using the MTT Cell Count kit (Nakalai Tesque, Kyoto, Japan). Cell migration was measured with the 96-well BME Cell-Invasion assay according to the manufacturer's instructions (Trevigen, Inc., MD, USA). Invasiveness was assayed by the three-dimensional Matrigel culture method $(23,24)$ as follows: a total of $8 \times 10^{5}$ transfected cells were suspended in $200 \mu 1$ Matrigel (Corning Inc., New York, NY, USA). A 150- $\mu 1$ drop of Matrigel mixture containing transfected cells was polymerized on the bottom of a 6-well microplate and incubated in $2 \mathrm{ml}$ medium for $48 \mathrm{~h}$. Photographs were acquired by fixed-point observation at X40 magnification under a BZ-X700 microscope (Keyence, Osaka, Japan). Invasiveness was assessed by the distance of cell migration. The distance of cell migration represented the length from the gel surface to the migratory front of cells that had invaded out of the gel. 
Table I. Clinical characteristics of patients with OSCC patients.

\begin{tabular}{|c|c|c|c|c|}
\hline & \multicolumn{2}{|c|}{ Primary OSCC } & \multicolumn{2}{|c|}{ Metastatic OSCC } \\
\hline & Total & $\begin{array}{l}\text { Lymph node- } \\
\text { negative } \\
(n=41)\end{array}$ & $\begin{array}{l}\text { Lymph node- } \\
\text { positive } \\
(\mathrm{n}=30)\end{array}$ & $\begin{array}{l}\text { Metastatic } \\
\text { lymph node } \\
(n=28)\end{array}$ \\
\hline \multicolumn{5}{|l|}{ Age } \\
\hline$<60$ & 18 & 7 & 11 & 9 \\
\hline$\geq 60$ & 53 & 34 & 19 & 19 \\
\hline \multicolumn{5}{|l|}{ Sex } \\
\hline Male & 47 & 24 & 23 & 21 \\
\hline Female & 24 & 17 & 7 & 7 \\
\hline \multicolumn{5}{|l|}{ Primary } \\
\hline \multicolumn{5}{|l|}{ lesion } \\
\hline Tongue & 33 & 20 & 13 & 12 \\
\hline Gum & 23 & 15 & 8 & 7 \\
\hline $\begin{array}{l}\text { Buccal } \\
\text { mucosa }\end{array}$ & 7 & 4 & 3 & 3 \\
\hline Other & 8 & 2 & 6 & 6 \\
\hline \multicolumn{5}{|c|}{ pTNM stage } \\
\hline I-II & 28 & 28 & 0 & 0 \\
\hline III-IV & 43 & 13 & 30 & 28 \\
\hline
\end{tabular}

pTNM, pathological tumor node metastasis.

Identification of candidate genes regulated by miR-205-5p. Possible miR-205-5p targets were identified by bioinformatics analysis with the TargetScan algorithm and integrated analysis across data for human cancer-cell lines and mRNA microarray data to identify miRNAs whose expression correlated with the inverse expression of the mRNA targets predicted in silico.

Plasmid construction and dual-luciferase reporter assay. Partial wild-type sequences of the TIMP-2 3'-UTR or those with a deleted miR-205-5p target sites (positions 590-596 of the TIMP-2 3'-UTR) were inserted between the XhoI-PmeI restriction sites in the 3'-UTR of hRluc gene in the psiCHECK-2 vector (Promega, Madison, WI, USA). The protocol for vector construction was as previously described $(22,25)$. The synthesized DNA was cloned into the psiCHECK-2 vector. The HSC3 cells were transfected with $50 \mathrm{ng}$ of the vector and $50 \mathrm{nM}$ miR-205-5p mimic using Lipofectamine 3000 (Invitrogen). The activities of firefly and Renilla luciferases in cell lysates were determined using a Dual-luciferase Reporter assay system (Promega). Normalized data were calculated as the ratio Renilla/firefly luciferase activities.

Western blot analysis. The cells were incubated at $37^{\circ} \mathrm{C}$ for $72 \mathrm{~h}$ after transfection, and then cell lysates were prepared. In addition, the culture media were centrifuged at 1,000 rpm and the supernatants were collected. The supernatants were inspissated with a Vivaspin2-10K (GE Healthcare UK Ltd., Little Chalfont, UK) according to the manufacturer's instructions. Subsequently, $15 \mu \mathrm{g}$ of protein lysates were separated on Mini-PROTEIN TGX Gels (Bio-Rad, Hercules, CA, USA) and then transferred onto Trans-Blot Turbo Mini PVDF membranes (Bio-Rad). The membranes were probed with rabbit anti-TIMP-2 antibodies (1:1,000; cat. no. 5738; Cell Signaling Technology, Danvers, MA, USA), rabbit MT1-MMP antibodies (1:1,000; cat. no. ab51074; Abcam, Cambridge, UK), and goat anti-actin antibodies (1:1000; cat. no. sc1615; Santa Cruz Biotechnology, Inc., Dallas, TX, USA) overnight at $4^{\circ} \mathrm{C}$. Proteins were detected with ChemiLumi One Super (Nakalai Tesque), and images of the blots were obtained with a ChemiDoc XRS Plus system (Bio-Rad).

Gelatin zymography. MMP-2 enzyme activity was analyzed in the cell supernatants by gelatin zymography using the Gelatin Zymography kit (Cosmo Bio Co., Ltd., Tokyo, Japan) according to the manufacturer's instructions. Images were obtained using the ChemiDoc XRS Plus system (Bio-Rad).

MMP-2 activity assay. The enzymatic activated MMP-2 content and total MMP-2 in the cell supernatants were determined using the QuickZyme Human MMP-2 Activity assay (QuickZyme BioSciences, Leiden, The Netherlands) according to the manufacturer's instructions.

Statistical analysis. Data for in vitro experiments were evaluated using the Student's t-test. Associations among more than 3 variables and numerical values were analyzed using the Kruskal-Wallis test with the Steel-Dwass test. The cut-off value of miR-205-5p expression in primary OSCC tissues were evaluated by the receiver operating characteristic (ROC) curve analysis. The analysis of disease-specific survival was performed by the Kaplan-Meier method and compared using the log-rank test. Correlations between miR-205-5p and TIMP-2 expression were evaluated by Spearman's rank test. All statistical analyses were performed using JMP version 11 software. A P-value $<0.05$ was considered to indicate a statistically significant difference.

\section{Results}

miR-205-5p expression in OSCC cell lines and clinical specimens. We evaluated miR-205-5p expression in 71 primary OSCC specimens, 28 metastatic lymph node specimens (patient characteristic shown in Table I), and in the HSC3 and SAS cell lines. We found that miR-205-5p expression was significantly lower in the primary OSCC tissue samples than in normal oral mucosa tissue samples, and that the expression was even lower in the metastatic lymph node tissue samples than in the primary OSCC tissue samples (Fig. 1A). On the other hand, the miR-205-5p expression was relatively low in primary OSCC tissue with metastasis compared to that without metastasis $(\mathrm{P}=0.103)$. Furthermore, we evaluated the power of miR-205-5p as the biomarker of prognosis prediction. We determined the cut-off value $(=3.74)$ of miR-205-5p in 71 primary OSCC tissue by receiver operating characteristic (ROC) curve analysis and analyzed disease-specific survival by the Kaplan-Meier method and compared using the log-rank test. The disease survival time was calculated from the date of the patient's first visit. None of the 71 OSCC 

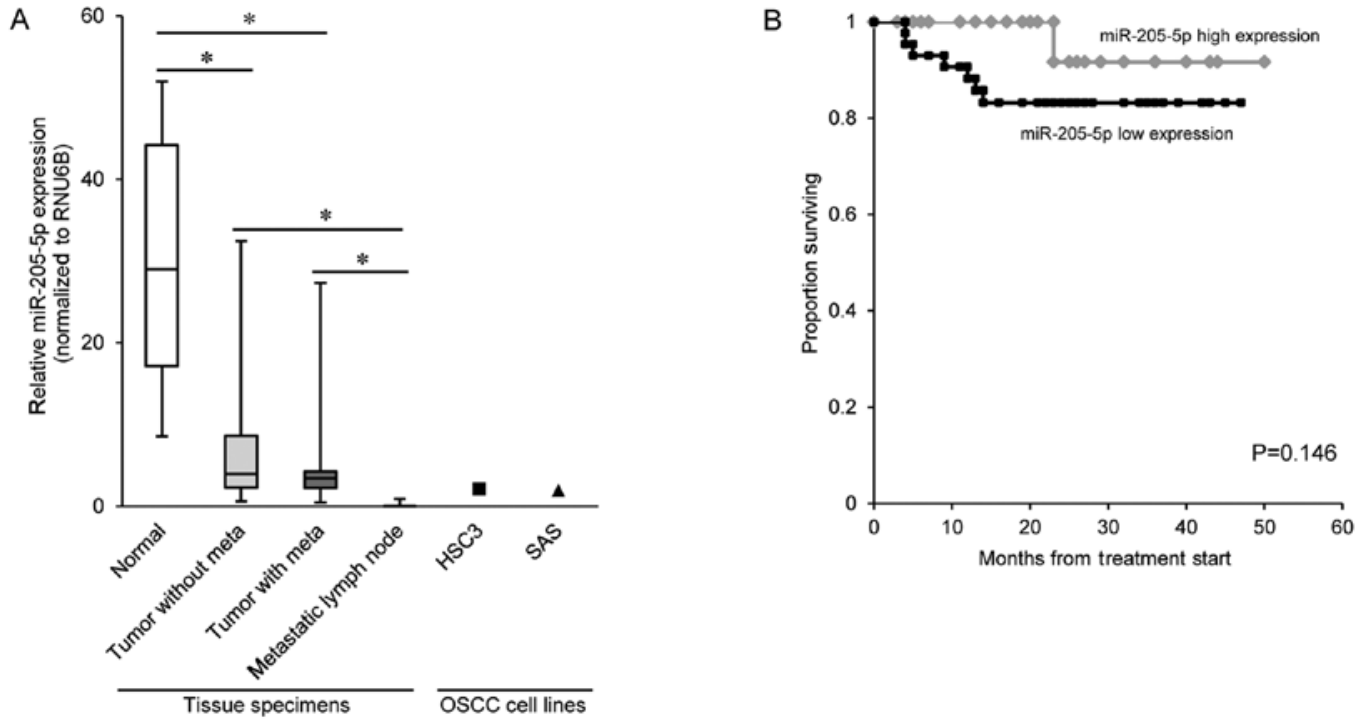

Figure 1. miR-205-5p expression in OSCC cell lines and clinical specimens. (A) Clinical specimens were classified as tissue with normal epithelia (n=6), primary tumor tissue without metastatic lymph node $(\mathrm{n}=41)$, primary tumor tissue with metastatic lymph node $(\mathrm{n}=30)$, or metastatic lymph node tissue $(\mathrm{n}=28)$, The HSC3 and SAS cell lines were used to analyze miRNA expression and function. RNU6B was used for normalization. Box-and-whisker plots represent data from clinical specimens. Middle line, median; lower whisker, minimum; upper whisker, maximum. Shapes representing cell lines indicate the median. ('P<0.05). (B) The 71 OSCC patients were divided into 2 groups (miR-205-5p high or low expression) according to the miR-205-5p expression in the primary OSCC tissue. Kaplan-Meier curves for overall survival in 29 subjects with a high miR-205-5p expression and 42 subjects with a low miR-205-5p expression. The difference between these 2 groups was determined by the log-rank test.
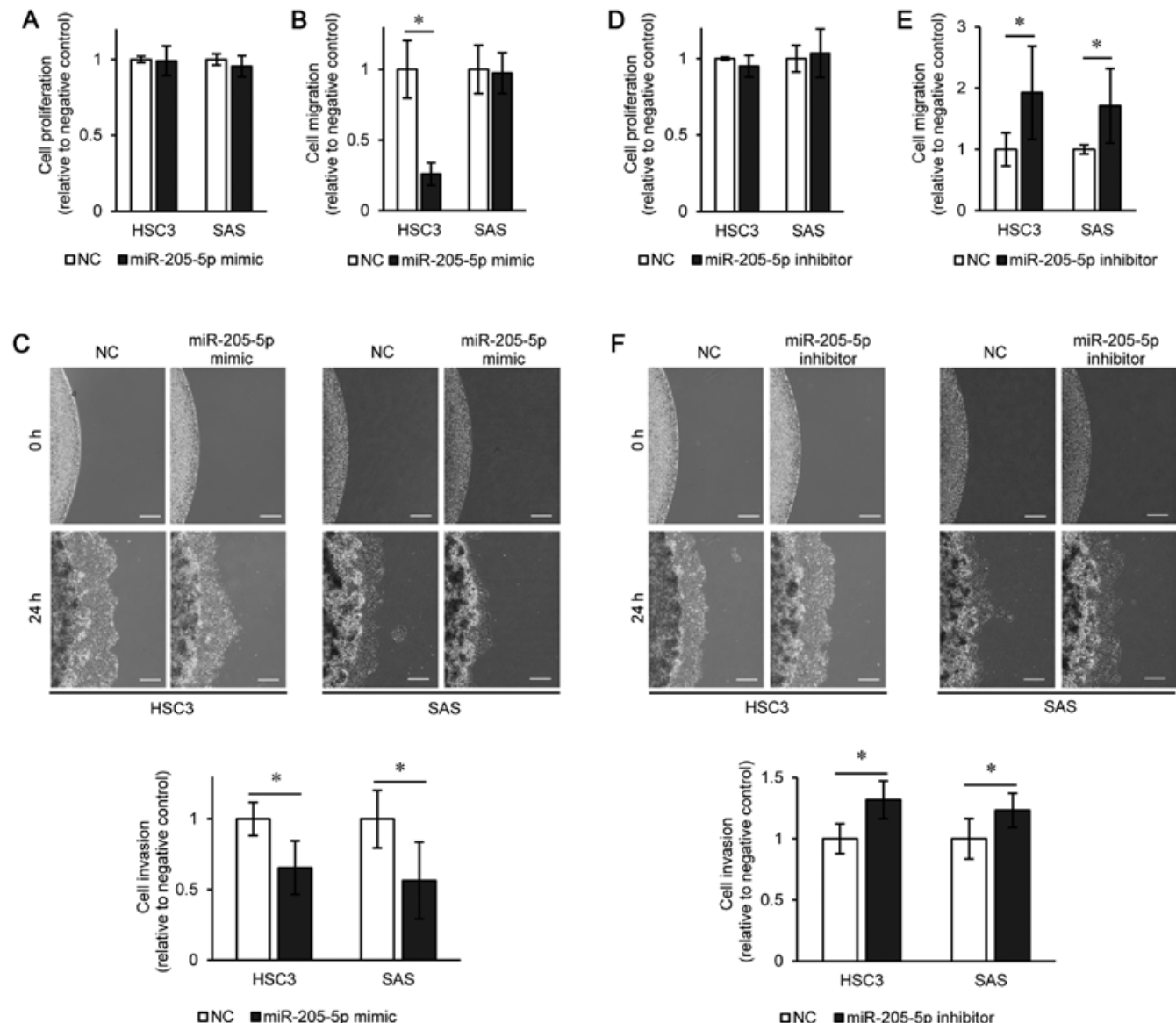

Figure 2. Effect of miR-205-5p mimic or inhibitor on OSCC cell proliferation, migration and invasiveness. HSC3 and SAS cells were transfected with $50 \mathrm{nM}$ miR-205-5p mimic or inhibitor or a negative control. (A and D) Cells were cultured for $24 \mathrm{~h}$ after transfection, and then assayed for proliferation. The value for the negative control (NC) was set to 1; bars represent the average of 6 wells per treatment. Error bars indicate the means \pm SD ("P<0.05). (B and E) The cells were cultured for $48 \mathrm{~h}$ after transfection and then assayed for migration. The value for negative NC was set to 1; bars represent the average of 6 wells per treatment. Error bars indicate the means $\pm \mathrm{SD}\left({ }^{*} \mathrm{P}<0.05\right)$. (C and F) The cells were cultured for $48 \mathrm{~h}$ after transfection and then assayed for invasive activity as described in the Materials and methods. The value for NC was set to 1; bars represent the average of 6 wells per treatment, and error bars indicate the means $\pm \mathrm{SD}\left({ }^{*} \mathrm{P}<0.05\right)$. Scale bar in images, $500 \mu \mathrm{m}$. 
Table II. miR-205-5p candidate target genes.

\begin{tabular}{lclcc}
\hline Gene symbol & Representative transcript & Gene name & Conserved & Poorly conserved \\
\hline TIMP-2 & NM_003255 & Metallopeptidase inhibitor 2 & 0 & 1 \\
MMP-16 & NM_005941 & Matrix metallopeptidase 16 & 0 & 1 \\
MMP-19 & NM_002429 & Matrix metallopeptidase 19 & 0 & 1 \\
\hline
\end{tabular}
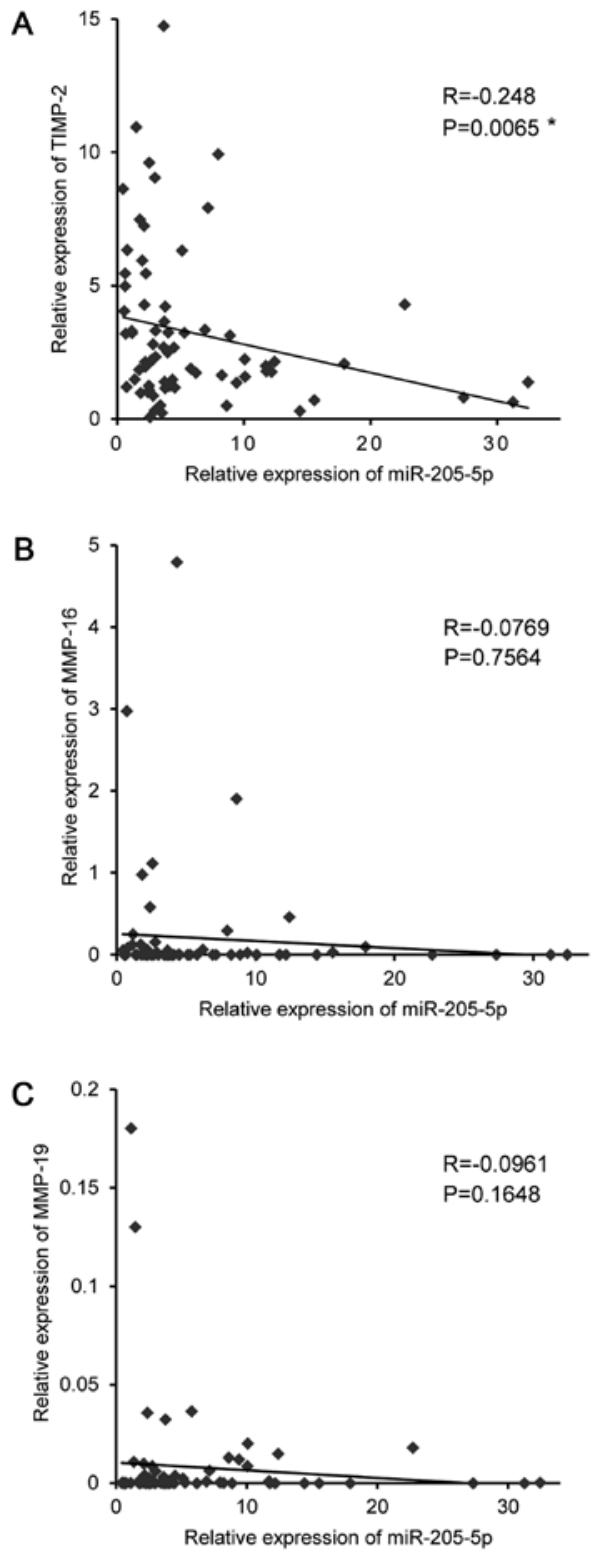

Figure 3. Negative correlation between miR-205-5p and TIMP-2 mRNA expression in OSCC specimens. (A-C) TIMP-2, MMP-16, MMP-19 and miR-205-5p expression levels were analyzed by RT-qPCR as described in the Materials and methods. TIMP-2/MMP-16/MMP-19 and miR-205-5p were normalized to GAPDH and RNU6B, respectively. P-values and $\mathrm{R}$ values were calculated using a Spearman's correlation test $\left({ }^{*} \mathrm{P}<0.01\right)$.

patients received pre-operative treatment. Therefore, 'the time of first visit' roughly equaled 'the time of treatment start'. There was no difference significant between the miR-205-5p high expression group and the miR-205-5p low expression group (Fig. 1B).
Effect of miR-205-5p mimic or inhibitor on cell proliferation, migration and invasion. Proliferation was not suppressed in the HSC3 or SAS cells transfected with miR-205-5p mimic compared to the negative control (Fig. 2A). HSC3 cell migration was significantly suppressed by transfection with miR-205-5p mimic compared to the negative control; however, SAS cell migration was not affected (Fig. 2B). The invasiveness of both the HSC3 and SAS cells was significantly suppressed following transfection with miR-205-5p mimic compared to the negative control (Fig. 2C). The number of cells that had invaded out of the gel was almost proportional to the migration distance. On the other hand, no significant differences in the proliferation of the HSC 3 and SAS cell transfected with miR-205-5p inhibitor were observed compared to the negative control (Fig. 2D). Both migration and invasiveness were significantly enhanced in the HSC3 and SAS cells transfected with miR-205-5p inhibitor as compared to the negative control (Fig. 2E and F).

Identification of miR-205-5p target genes in OSCC. We screened the TargetScan database for possible target genes containing a putative miR-205-5p-binding site in their 3'-UTR, and identified 5,974 candidate genes that may be regulated by $\mathrm{miR}-205-5 \mathrm{p}$. From these, we selected genes related to TIMPs and MMPs, and identified 3 candidate miR-205-5p targets (Table II). Among these candidates, we focused on the TIMP-2 gene, as Spearman's rank test revealed a significant negative correlation between the mRNA expression of TIMP-2 and that of miR-205-5p in OSCC specimens (Fig. 3).

TIMP-2 is a direct target of miR-205-5p in OSCC cells. The TargetScan database revealed one putative, poorly conserved miR-205-5p-binding site in the 3'-UTR of TIMP-2 (position 590-596) (Fig. 4A). We thus examined TIMP-2 expression in miR-205-5p mimic-transfected OSCC cells, and found that the TIMP-2 mRNA and protein expression levels were suppressed in the cells transfected with miR-205-5p mimic compared to the negative control (Fig. 4B and C). To determine whether TIMP-2 mRNA contains a target site for miR-205-5p, we conducted luciferase reporter assays using the HSC3 and SAS cells. The TargetScan database revealed that there was one putative miR-205-5p binding site in the TIMP-2 3'-UTR (position 590-596). We used vectors encoding either a partial wild-type sequence (including the predicted miR-205-5p target site) or deletion of the seed sequence of the 3'-UTR of TIMP-2 mRNA. We found that the luciferase activity was significantly suppressed by co-transfection with miR-205-5p mimic and the vector carrying the wild-type 3'-UTR of TIMP-2 (Fig. 4D). 

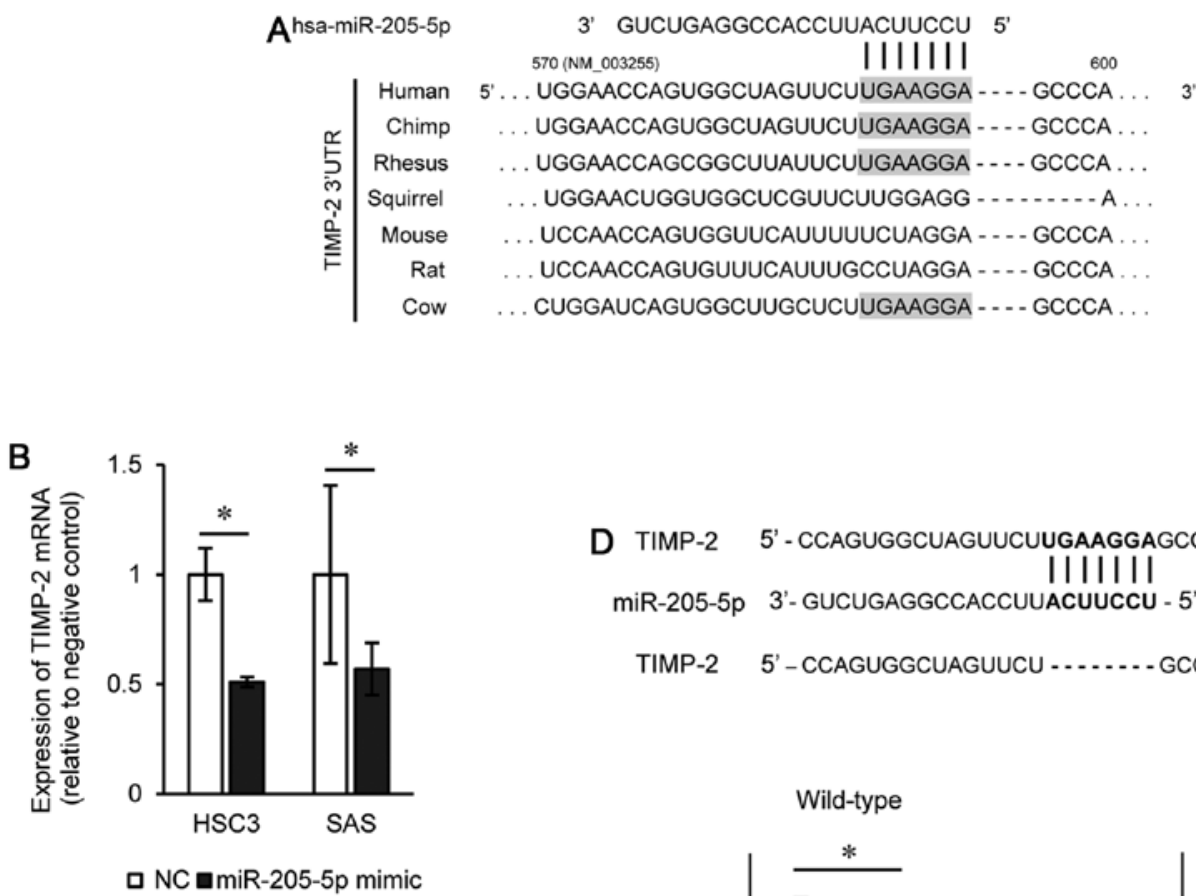

\author{
D TIMP-2 5' - CCAGUgGCUAGUUCUUGAaggagCCCA - 3' Wild-type \\ IIIIIII \\ miR-205-5p 3'- GUCUGAGGCCACCUUACUUCCU - 5 \\ TIMP-2 5' -CCAgUgGCUAGUUCU -......... - GCCCA - 3' Deletion-type
}
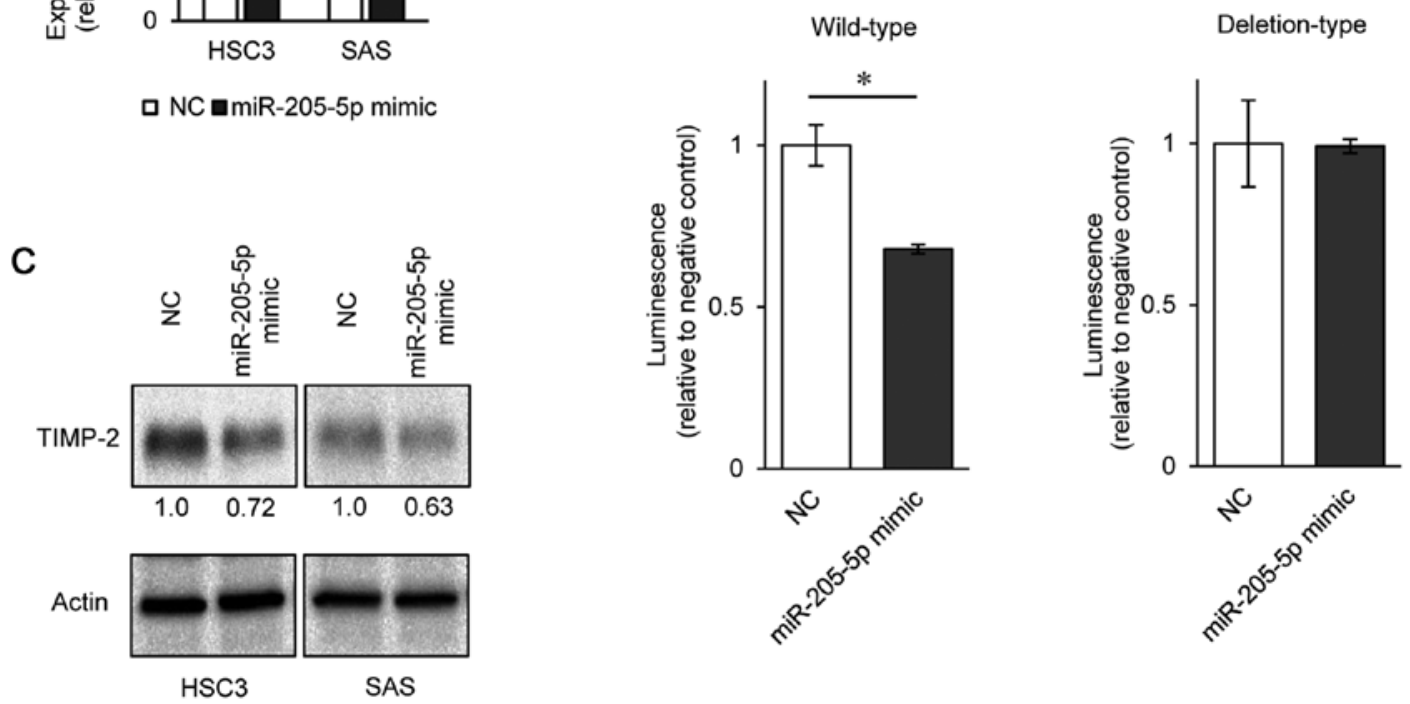

Figure 4. TIMP-2 is a direct target of miR-205-5p. (A) Schematic showing the conservation of a putative miR-205-5p target site in the 3'-UTR of TIMP-2. (B) TIMP-2 mRNA levels in HSC3 and SAS cells transfected with miR-205-5p mimic or negative control. TIMP-2 was normalized to GAPDH. The expression of the negative control (NC) was set to 1; bars represent the average of 6 wells per treatment, and the error bars are SD ("P $<0.05$ ). (C) Western blot showing the TIMP-2 levels in HSC3 and SAS cells transfected with miR-205-5p mimic or negative control. Actin was used as a loading control. (D) Luciferase reporter assays were performed using vectors that included (wild-type) or lucked (deletion type) the wild-type sequences of the putative miR-205-5p target site. Renilla luciferase assays were normalized to firefly luciferase values. The luminescence for NC was set to 1 ; bars represent the average of 6 wells per treatment, and error bars indicate the means $\pm \mathrm{SD}\left({ }^{*} \mathrm{P}<0.05\right)$.

Effect of TIMP-2 silencing on OSCC cell lines. We then investigated the functions of TIMP-2 in HSC3 and SAS cells by loss-of-function experiments using si-TIMP-2 transfection. The results of western blot analysis and RT-qPCR indicated that si-TIMP-2 effectively downregulated TIMP-2 expression in both cell lines (Fig. 5A and B). The invasiveness of both the HSC3 and SAS cells was suppressed following transfection with si-TIMP-2 compared to the negative control (Fig. 5C).

Suppression of TIMP-2 reduces the activation of pro-MMP-2 via the TIMP-2/MT1-MMP complex. To investigate whether reducing TIMP-2 expression affects the activation of pro-MMP-2 by MT1-MMP, we used western blot analysis or gelatin zymography to measure the expression of TIMP-2, MT1-MMP, pro-MMP-2 and active-MMP-2 in HSC3 and SAS cells transfected with miR-205-5p mimic or si-TIMP-2. The results of western blot analysis revealed that TIMP-2 expression was decreased in the supernatant of both cells transfected with miR-205-5p mimic or si-TIMP-2 compared to the negative control, and that MT1-MMP expression was comparable in both cells transfected with miR-205-5p mimic, si-TIMP-2, or negative control (Fig. 6A). We then measured pro-MMP-2 activation in the cell supernatants (see Materials and methods). MMP-2 activity assay revealed that the rate of pro-MMP-2 activation was significantly lower in both cells transfected with miR-205 mimic or si-TIMP-2 than in those transfected with the negative control (Fig. 6B). The rate of pro-MMP-2 activation represented the percentage of active-MMP-2 to total MMP-2 (pro-MMP-2 + active-MMP-2). The results of gelatin zymography revealed that activated pro-MMP-2 (active-MMP-2) was decreased in both cells transfected with miR-205-5p mimic compard with those transfected with the negative control (Fig. 6C). 

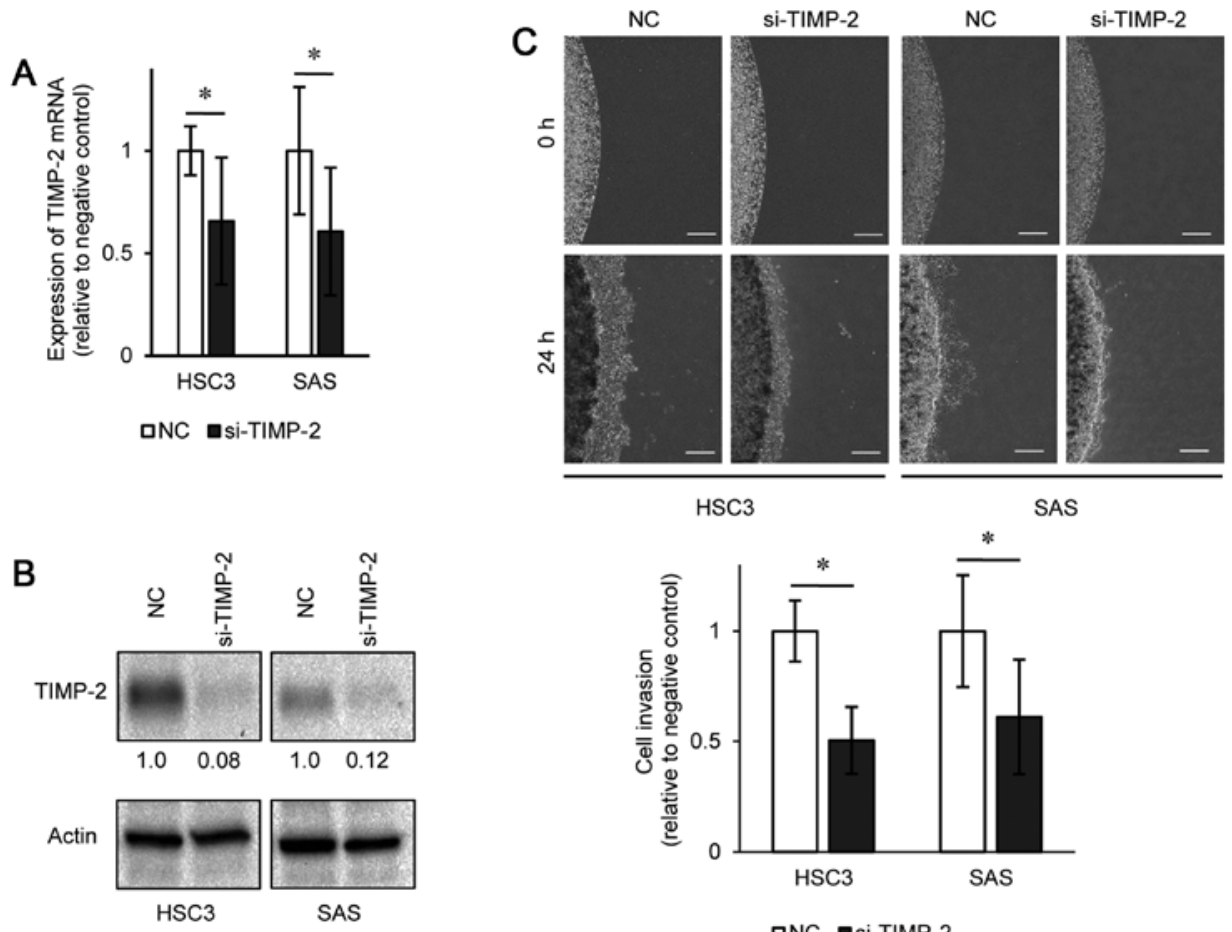

aNC घSi-TIMP-2

Figure 5. Effect of si-TIMP-2 transfection on OSCC cell lines. (A) TIMP-2 mRNA levels, normalized to GAPDH, in HSC3 and SAS cells transfected with si-TIMP-2 $(25 \mathrm{nM})$ or negative control. The value for the negative control $(\mathrm{NC})$ was set to 1; bars represent the average of 6 wells per treatment, and error bars indicate the means \pm SD ("P<0.05). (B) Representative western blots showing the TIMP-2 levels in HSC3 and SAS cells following transfection with si-TIMP-2 or negative control. Actin was used as a loading control. (C) Cells were cultured for $48 \mathrm{~h}$ after transfection, and then assayed for invasiveness (see Materials and methods). The value for the negative control was set to 1 ; bars represent the average of 6 wells per treatment, and error bars indicate the means \pm SD $($ " $P<0.05)$. Scale bars, $500 \mu \mathrm{m}$.
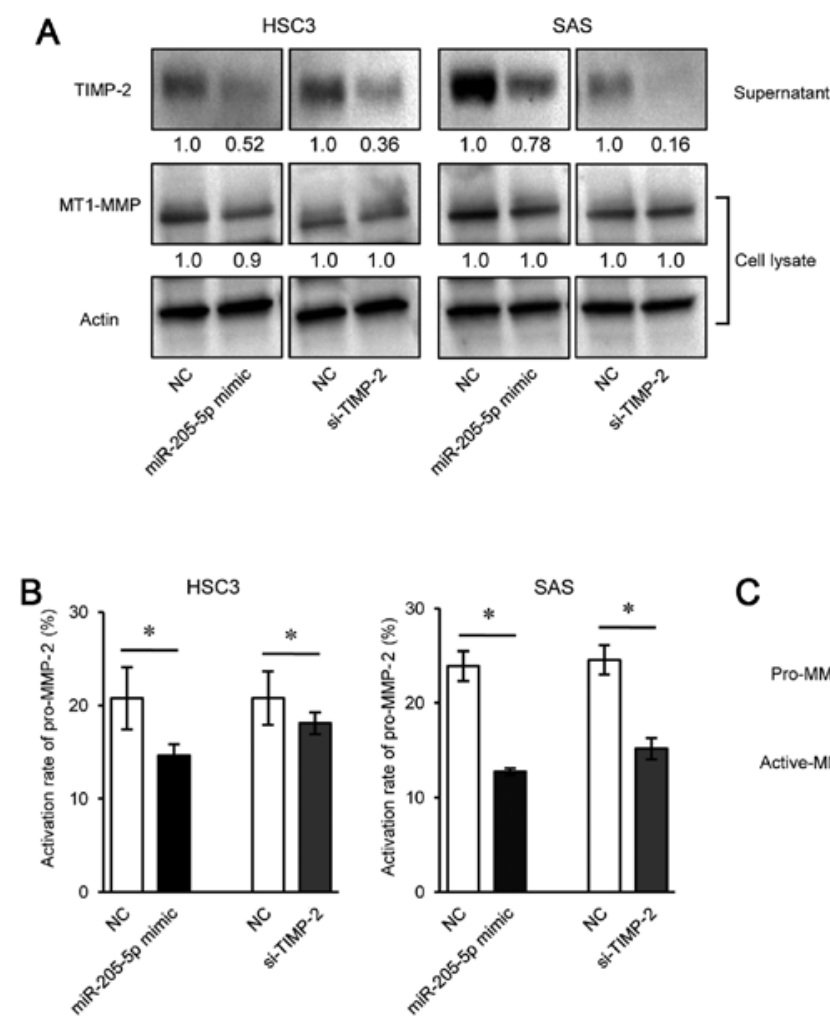

C

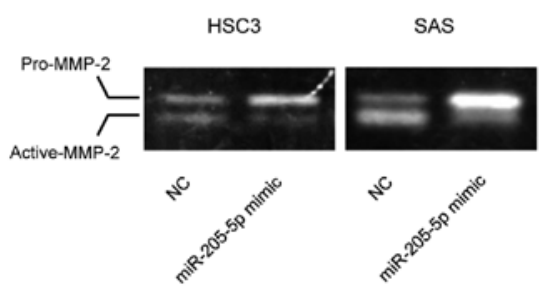

Figure 6. TIMP-2 suppression in OSCC cells reduces the activation of pro-MMP-2 via the TIMP-2/MT1-MMP complex. HAC3 and SAS cells were transfected with miR-205-5p mimic, si-TIMP-2, or negative control. (A) Cells were cultured in FBS-free medium for $48 \mathrm{~h}$, the contents of TIMP- 2 in the culture supernatant and of MT1-MMP in the cells were then analyzed by western blot analysis. Actin was used as a loading control. (B) Cells were cultured in FBS-free medium for $48 \mathrm{~h}$, and the amount of activated pro-MMP-2 in the supernatant was then analyzed as described in the Materials and methods. The value for negative control (NC) was set to 1; bars represent the average of 4 wells per treatment, and error bars indicate the means \pm SD ("P<0.05). (C) Cells were cultured in FBS-free medium for $48 \mathrm{~h}$, and the MMP-2 enzyme activity in the supernatant was then analyzed by gelatin zymography. 


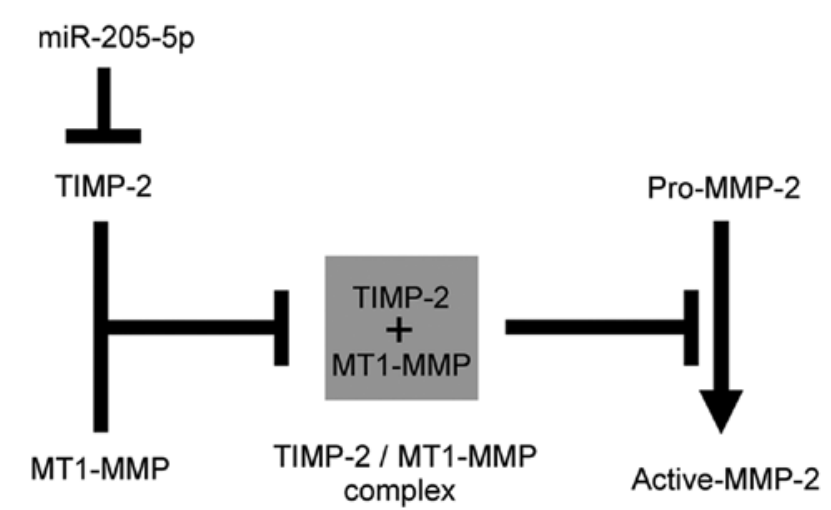

Figure 7. Pathway through which miR-205-5p regulates TIMP-2 to suppress pro-MMP-2 activation. miR-205-5p suppresses TIMP-2 expression, reducing the amount of TIMP-2/MT1-MMP complex on the cell membrane. Thus, pro-MMP-2 activation mediated by the membrane-bound TIMP-2/MT1-MMP complex is suppressed.

\section{Discussion}

There is growing evidence to indicate the involvement of miRNAs in several biological processes, including human oncogenesis and metastasis (26); these include miR-29c, miR-335 and miR-375 (27,28). Studies have shown that miR-155-5p is downregulated in OSCC tissue, and that a low miR-155-5p level correlates with a poor prognosis (14). Therefore, the investigation of miRNA expression signatures in cancer specimens is an important research avenue. In the present study, we focused on miR-205-5p, and yielded 3 major findings: first, that miR-205-5p acted as a tumor suppressor in OSCC cells (Figs. 1 and 2); second, that miR-205-5p directly suppressed TIMP-2 expression in OSCC cells (Fig. 4); and third, that by suppressing TIMP-2 expression, miR-205-5p inhibited pro-MMP-2 activation (Fig. 6).

In this study, we observed that miR-205-5p expression was significantly downregulated in primary OSCC tissues compared to normal oral mucosa tissue, and that the expression was even lower in metastatic lymph node tissue than in primary OSCC tissue (Fig. 1A). We also demonstrated that transfection with miR-205-5p suppressed the invasiveness of both the HSC3 and SAS cells (Fig. 2C). These results suggest that miR-205-5p acts as a tumor suppressor miRNA in OSCC, and that a reduced miR-205-5p expression may significantly contribute to OSCC invasion and metastasis. Recent studies have indicated that miR-205-5p is downregulated in several types of cancer, including breast, prostate and bladder cancer (29-31), and that miR-205-5p suppresses several tumor types by targeting oncogenes (16,19,32-34). Other studies have shown that miR-205-5p suppresses cell invasiveness in several types of cancer $(18,35)$. The role of miR-205-5p in suppressing cell invasion has also been confirmed in in vivo models of breast cancer (30). These findings are consistent with those of the present study. By contrast, Kaplan-Meier analysis revealed that there was no difference between the miR-205-5p high expression group and the miR-205-5p low expression group (Fig. 1B). A recent study also demonstrated that a low expression of miR-205 was associated with a poor prognosis in cervical cancer (36). Another previous study demonstrated that a low expression of both miR-205 and let-7d was associ- ated with a poor prognosis in head and neck squamous-cell carcinoma (HNSCC); however, this association was not observed with a low expression of miR-205 only (37). To reveal the correlation between the expression of miR-205-5p and disease-specific survival in OSCC, further research is required, examining more cases of OSCC.

A single miRNA can regulate several protein-coding genes. Indeed, bioinformatics analyses have shown that at least $30-60 \%$ of the protein-coding genes in the human genome are regulated by miRNAs (38). Therefore, a reduced expression of tumor suppressor miRNAs may cause an overexpression of oncogenic genes in cancer cells.

In this study, we identified TIMP-2 as a target of miR-205-5p. Other recent studies have shown that TIMP-2 is an oncogenic factor in several types of cancer $(39,40)$. Our data demonstrated a significant negative correlation between the mRNA expression of TIMP-2 and that of miR-205-5p in OSCC tissues ( $R=-0.248, P=0.0065$ ) (Fig. 3). Furthermore, our luciferase reporter assays confirmed that miR-205-5p miRNA directly binds the 3'-UTR of TIMP-2 (Fig. 4D). To the best of our knowledge, this is the first study to show that TIMP-2 is directly regulated by miR-205-5p in OSCC cells.

TIMP-2 is known to regulate MMPs through its enzymatic activity. The data from the present study demonstrated that silencing TIMP-2 suppressed the invasiveness of OSCC cells (Fig. 5), suggesting that TIMP-2 promotes OSCC tumor progression. Recent immunohistochemical analyses have identified an elevated TIMP-2 expression as an indicator of aggressive behavior and a poor prognosis in patients with HNSCC (41), and demonstrated a strong correlation between a marked TIMP-2 expression and lymph-node metastasis, as well as a poor prognosis, in the early stages of OSCC (41-43). These data are consistent with the findings of the present study, indicating that TIMP-2 may promote OSCC tumor progression. Recent studies have shown that TIMP-2 promotes tumor progression via the activation of MMP-2, which is mediated by MT1-MMP $(9,10)$. TIMP-2 was long thought to suppress tumor invasion by inhibiting MMP-2 in general, and when overexpressed, TIMP-2 inhibited MMP-2 in vitro. However, according to a model of cell-mediated MMP-2 activation, pro-MMP-2 binds TIMP-2 in a complex with MT1-MMP on the cell surface, forming a pro-MMP-2/TIMP-2/MT1-MMP ternary complex. In this case, pro-MMP-2 is activated by the adjacent MT1-MMP that is not bound to TIMP-2 $(9,10,44)$. In the present study, although we observed that TIMP-2 expression was decreased in the supernatant of miR-205-5p mimic-transfected HSC3 and SAS cells, MT1-MMP expression was not affected (Fig. 6A). In addition, pro-MMP-2 was activated at a lower rate in miR-205-5p mimic-transfected cells (Fig. 6B and $C$ ). These results suggest that miR-205-5p suppresses pro-MMP-2 activation by regulating TIMP-2, as illustrated in Fig. 7. Recent studies have shown that TIMP-2 enhances pro-MMP-2 activation via MT1-MMP in vivo (45-49), and that upregulating TIMP-2 promotes MMP- 2 activation and the invasiveness of glioma cells (47). Another study demonstrated that the ratio of MMP-2 activation strongly correlated with TIMP-2 expression in SCC of the tongue (42). These findings are consistent with those of the present study. Pro-MMP-2 activation depends on the local TIMP-2 concentration; this activation occurs at a low concentration of TIMP-2 relative 
to MT1-MMP, leaving sufficient inhibitor-free MT1-MMP to initiate the activation of pro-MMP-2 $(50,51)$. On the other hand, high TIMP-2 levels inhibit MMP-2 activation by blocking all of the available MT1-MMP molecules (52). In the present study, the amount of TIMP-2 secreted into the culture medium appeared to be appropriate for regulating MMP-2 activation in HSC 3 and SAS cells. To further determine the role of miR-205-5p in the regulation of TIMP-2 expression in OSCC, further studies using animal models are warranted.

In conclusoin, the present study demonstrates that miR-205-5p functions as a tumor suppressor in OSCC, that miR-205-5p directly regulates TIMP-2 expression, and that miR-205-5p suppresses pro-MMP-2 activation by regulating TIMP-2 expression in OSCC cells (Fig. 7).

This identification of the tumor suppressor role of miR-205-5p in OSCC may have significant therapeutic potential. Currently, RNA interference is being implemented as a gene-specific approach in molecular medicine. Thus, it may be possible to use miR-205-5p to regulate specific genes related to tumor progression as a novel therapeutic approach to the treatment of OSCC.

\section{Acknowledgements}

This study was supported by JSPS KAKENHI (grant nos. 25893024 and 15H05038), and was partly supported by the Extramural Collaborative Research Grant of the Cancer Research Institute, Kanazawa University.

\section{Competing interests}

The authors declare that they have no competing interests.

\section{References}

1. Bhattacharya A, Roy R, Snijders AM, Hamilton G, Paquette J, Tokuyasu T, Bengtsson H, Jordan RCK, Olshen AB, Pinkel D, et al: Two distinct routes to oral cancer differing in genome instability and risk for cervical node metastasis. Clin Cancer Res 17: 7024-7034, 2011

2. Ferlito A, Rinaldo A, Robbins KT, Leemans CR, Shah JP, Shaha AR, Andersen PE, Kowalski LP, Pellitteri PK, Clayman GL, et al: Changing concepts in the surgical management of the cervical node metastasis. Oral Oncol 39: 429-435, 2003.

3. Greenberg JS, El Naggar AK, Mo V, Roberts D and Myers JN: Disparity in pathologic and clinical lymph node staging in oral tongue carcinoma. Implication for therapeutic decision making. Cancer 98: 508-515, 2003.

4. Shingaki S, Takada M, Sasai K, Bibi R, Kobayashi T, Nomura T and Saito C: Impact of lymph node metastasis on the pattern of failure and survival in oral carcinomas. Am J Surg 185: 278-284, 2003.

5. Woolgar JA, Rogers SN, Lowe D, Brown JS and Vaughan ED: Cervical lymph node metastasis in oral cancer: The importance of even microscopic extracapsular spread. Oral Oncol 39: 130-137, 2003.

6. Wikner J, Gröbe A, Pantel K and Riethdorf S: Squamous cell carcinoma of the oral cavity and circulating tumour cells. World J Clin Oncol 5: 114-124, 2014.

7. Bhuvarahamurthy V, Kristiansen GO, Johannsen M, Loening SA, Schnorr D, Jung K and Staack A: In situ gene expression and localization of metalloproteinases MMP1, MMP2, MMP3, MMP9, and their inhibitors TIMP1 and TIMP2 in human renal cell carcinoma. Oncol Rep 15: 1379-1384, 2006.

8. Kusukawa J,Sasaguri Y,Shima I, Kameyama T and Morimatsu M: Expression of matrix metalloproteinase-2 related to lymph node metastasis of oral squamous cell carcinoma. A clinicopathologic study. Am J Clin Pathol 99: 18-23, 1993.
9. Imai K, Ohuchi E, Aoki T, Nomura H, Fujii Y, Sato H, Seiki M and Okada Y: Membrane-type matrix metalloproteinase 1 is a gelatinolytic enzyme and is secreted in a complex with tissue inhibitor of metalloproteinases 2. Cancer Res 56: 2707-2710, 1996.

10. Strongin AY, Collier I, Bannikov G, Marmer BL, Grant GA and Goldberg GI: Mechanism of cell surface activation of 72-kDa type IV collagenase. Isolation of the activated form of the membrane metalloprotease. J Biol Chem 270: 5331-5338, 1995.

11. Brodersen $\mathrm{P}$ and Voinnet $\mathrm{O}$ : Revisiting the principles of microRNA target recognition and mode of action. Nat Rev Mol Cell Biol 10: 141-148, 2009.

12. Fukumoto I, Koshizuka K, Hanazawa T, Kikkawa N, Matsushita R, Kurozumi A, Kato M, Okato A, Okamoto Y and Seki N: The tumor-suppressive microRNA-23b/27b cluster regulates the MET oncogene in oral squamous cell carcinoma. Int J Oncol 49: 1119-1129, 2016.

13. Fukumoto I, Hanazawa T, Kinoshita T, Kikkawa N, Koshizuka K, Goto Y, Nishikawa R, Chiyomaru T, Enokida H, Nakagawa M, et al: MicroRNA expression signature of oral squamous cell carcinoma: Functional role of microRNA-26a/b in the modulation of novel cancer pathways. Br J Cancer 112 . 891-900, 2015.

14. Baba O, Hasegawa S, Nagai H, Uchida F, Yamatoji M, Kanno NI, Yamagata K, Sakai S, Yanagawa T and Bukawa H: MicroRNA$155-5 \mathrm{p}$ is associated with oral squamous cell carcinoma metastasis and poor prognosis. J Oral Pathol 45: 248-255, 2016.

15. He S, Lai R, Chen D, Yan W, Zhang Z, Liu Z, Ding X and Chen Y: Downregulation of miR-221 inhibits cell migration and invasion through targeting methyl- $\mathrm{CpG}$ binding domain protein 2 in human oral squamous cell carcinoma cells. BioMed Res Int 2015: 751672, 2015.

16. Elgamal OA, Park JK, Gusev Y, Azevedo-Pouly AC, Jiang J, Roopra A and Schmittgen TD: Tumor suppressive function of mir-205 in breast cancer is linked to HMGB3 regulation. PLoS One 8: e76402, 2013.

17. Iorio MV, Casalini P, Piovan C, Di Leva G, Merlo A, Triulzi T, Ménard S, Croce CM and Tagliabue E: microRNA-205 regulates HER3 in human breast cancer. Cancer Res 69: 2195-2200, 2009.

18. Majid S, Dar AA, Saini S, Yamamura S, Hirata H, Tanaka Y, Deng G and Dahiya R: MicroRNA-205-directed transcriptional activation of tumor suppressor genes in prostate cancer. Cancer 116: 5637-5649, 2010.

19. Matsushima K, Isomoto H, Yamaguchi N, Inoue N, Machida H, Nakayama T, Hayashi T, Kunizaki M, Hidaka S, Nagayasu T, et al: miRNA-205 modulates cellular invasion and migration via regulating zinc finger E-box binding homeobox 2 expression in esophageal squamous cell carcinoma cells. J Transl Med 9: 30, 2011.

20. Wang G, Wang L, Sun S, Wu J and Wang Q: Quantitative measurement of serum microRNA-21 expression in relation to breast cancer metastasis in Chinese females. Ann Lab Med 35: 226-232, 2015.

21. Wei J, Zhang L, Li J, Zhu S, Tai M, Mason CW, Chapman JA, Reynolds EA, Weiner CP and Zhou HH: MicroRNA-205 promotes cell invasion by repressing TCF21 in human ovarian cancer. J Ovarian Res 10: 33, 2017.

22. Kinoshita T, Nohata N, Hanazawa T, Kikkawa N, Yamamoto N, Yoshino H, Itesako T, Enokida H, Nakagawa M, Okamoto Y, et al: Tumour-suppressive microRNA-29s inhibit cancer cell migration and invasion by targeting laminin-integrin signalling in head and neck squamous cell carcinoma. Br J Cancer 109: 2636-2645, 2013.

23. Lee GY, Kenny PA, Lee EH and Bissell MJ: Three-dimensional culture models of normal and malignant breast epithelial cells. Nat Methods 4: 359-365, 2007.

24. Sakr M, Takino T, Sabit H, Nakada M, Li Z and Sato H: miR-150-5p and miR-133a suppress glioma cell proliferation and migration through targeting membrane-type-1 matrix metalloproteinase. Gene 587: 155-162, 2016.

25. Nishikawa R, Goto Y, Kojima S, Enokida H, Chiyomaru T, Kinoshita T, Sakamoto S, Fuse M, Nakagawa M, Naya Y, et al: Tumor-suppressive microRNA-29s inhibit cancer cell migration and invasion via targeting LAMC1 in prostate cancer. Int $\mathrm{J}$ Oncol 45: 401-410, 2014.

26. Nelson KM and Weiss GJ: MicroRNAs and cancer: Past, present, and potential future. Mol Cancer Ther 7: 3655-3660, 2008.

27. Liu L, Bi N, Wu L, Ding X, Men Y, Zhou W, Li L, Zhang W, Shi S, Song Y, et al: MicroRNA-29c functions as a tumor suppressor by targeting VEGFA in lung adenocarcinoma. Mol Cancer 16: 50, 2017. 
28. Zhang JK, Li YS, Zhang CD and Dai DQ: Up-regulation of CRKL by microRNA-335 methylation is associated with poor prognosis in gastric cancer. Cancer Cell Int 17: 28, 2017.

29. Chen Z, Tang ZY, He Y, Liu LF, Li DJ and Chen X: miRNA-205 is a candidate tumor suppressor that targets ZEB2 in renal cell carcinoma. Oncol Res Treat 37: 658-664, 2014

30. Wu H, Zhu S and Mo YY: Suppression of cell growth and invasion by miR-205 in breast cancer. Cell Res 19: 439-448, 2009.

31. Wang N, Li Q, Feng NH, Cheng G, Guan ZL, Wang Y, Qin C, Yin CJ and Hua LX: miR-205 is frequently downregulated in prostate cancer and acts as a tumor suppressor by inhibiting tumor growth. Asian J Androl 15: 735-741, 2013.

32. Gregory PA, Bert AG, Paterson EL, Barry SC, Tsykin A, Farshid G, Vadas MA, Khew-Goodall Y and Goodall GJ: The miR-200 family and miR-205 regulate epithelial to mesenchymal transition by targeting ZEB1 and SIP1. Nat Cell Biol 10: 593-601, 2008.

33. Yue X, Wang P, Xu J, Zhu Y, Sun G, Pang Q and Tao R: MicroRNA-205 functions as a tumor suppressor in human glioblastoma cells by targeting VEGF-A. Oncol Rep 27: 1200-1206, 2012.

34. Adachi R, Horiuchi S, Sakurazawa Y, Hasegawa T, Sato K and Sakamaki T: ErbB2 down-regulates microRNA-205 in breast cancer. Biochem Biophys Res Commun 411: 804-808, 2011.

35. Zhang $\mathrm{H}$ and Fan Q: MicroRNA-205 inhibits the proliferation and invasion of breast cancer by regulating AMOT expression. Oncol Rep 34: 2163-2170, 2015.

36. Pang $\mathrm{H}$ and Yue X: miR-205 serves as a prognostic factor and suppresses proliferation and invasion by targeting insulin-like growth factor receptor 1 in human cervical cancer. Tumour Biol 39: 1010428317701308, 2017.

37. Childs G, Fazzari M, Kung G, Kawachi N, Brandwein-Gensler M, McLemore M, Chen Q, Burk RD, Smith RV, Prystowsky MB, et al: Low-level expression of microRNAs let-7d and miR-205 are prognostic markers of head and neck squamous cell carcinoma. Am J Pathol 174: 736-745, 2009.

38. Lewis BP, Burge CB and Bartel DP: Conserved seed pairing, often flanked by adenosines, indicates that thousands of human genes are microRNA targets. Cell 120: 15-20, 2005.

39. Grignon DJ, Sakr W, Toth M, Ravery V, Angulo J, Shamsa F, Pontes JE, Crissman JC and Fridman R: High levels of tissue inhibitor of metalloproteinase-2 (TIMP-2) expression are associated with poor outcome in invasive bladder cancer. Cancer Res 56: 1654-1659, 1996

40. Ree AH, Florenes VA, Berg JP, Maelandsmo GM, Nesland JM and Fodstad O: High levels of messenger RNAs for tissue inhibitors of metalloproteinases (TIMP-1 and TIMP-2) in primary breast carcinomas are associated with development of distant metastases. Clin Cancer Res 3: 1623-1628, 1997.

41. Ondruschka C, Buhtz P, Motsch C, Freigang B, Schneider-Stock R Roessner A and Boltze C: Prognostic value of MMP-2, -9 and TIMP-1,-2 immunoreactive protein at the invasive front in advanced head and neck squamous cell carcinomas. Pathol Res Pract 198: 509-515, 2002.
42. Yoshizaki T, Maruyama Y, Sato H and Furukawa M: Expression of tissue inhibitor of matrix metalloproteinase-2 correlates with activation of matrix metalloproteinase-2 and predicts poor prognosis in tongue squamous cell carcinoma. Int $\mathbf{J}$ Cancer 95: 44-50, 2001.

43. Katayama A, Bandoh N, Kishibe K, Takahara M, Ogino T, Nonaka $S$ and Harabuchi Y: Expressions of matrix metalloproteinases in early-stage oral squamous cell carcinoma as predictive indicators for tumor metastases and prognosis. Clin Cancer Res 10: 634-640, 2004.

44. Sato H, Takino T, Kinoshita T, Imai K, Okada Y, Stetler Stevenson WG and Seiki M: Cell surface binding and activation of gelatinase A induced by expression of membrane-type-1-matrix metalloproteinase (MT1-MMP). FEBS Lett 385: 238-240, 1996.

45. Hernandez-Barrantes S, Toth M, Bernardo MM, Yurkova M Gervasi DC, Raz Y, Sang QA and Fridman R: Binding of active $(57 \mathrm{kDa})$ membrane type 1-matrix metalloproteinase (MT1-MMP) to tissue inhibitor of metalloproteinase (TIMP)-2 regulates MT1-MMP processing and pro-MMP-2 activation. J Biol Chem 275: 12080-12089, 2000.

46. Bernardo MM and Fridman R: TIMP-2 (tissue inhibitor of metalloproteinase-2) regulates MMP-2 (matrix metalloproteinase-2) activity in the extracellular environment after pro-MMP-2 activation by MT1 (membrane type 1)-MMP. Biochem J 374: 739-745, 2003.

47. Lu KV, Jong KA, Rajasekaran AK, Cloughesy TF and Mischel PS: Upregulation of tissue inhibitor of metalloproteinases (TIMP)-2 promotes matrix metalloproteinase (MMP)-2 activation and cell invasion in a human glioblastoma cell line. Lab Invest 84: 8-20, 2004.

48. Kudo T, Takino T, Miyamori H, Thompson EW and Sato $\mathrm{H}$ : Substrate choice of membrane-type 1 matrix metalloproteinase is dictated by tissue inhibitor of metalloproteinase-2 levels. Cancer Sci 98: 563-568, 2007.

49. Nishida Y, Miyamori H, Thompson EW, Takino T, Endo Y and Sato H: Activation of matrix metalloproteinase-2 (MMP-2) by membrane type 1 matrix metalloproteinase through an artificial receptor for proMMP-2 generates active MMP-2. Cancer Res 68: 9096-9104, 2008.

50. Caterina JJ, Yamada S, Caterina NC, Longenecker G, Holmbäck K, Shi J, Yermovsky AE, Engler JA and BirkedalHansen $\mathrm{H}$ : Inactivating mutation of the mouse tissue inhibitor of metalloproteinases-2 (Timp-2) gene alters proMMP-2 activation. J Biol Chem 275: 26416-26422, 2000.

51. Wang Z, Juttermann R and Soloway PD: TIMP-2 is required for efficient activation of proMMP-2 in vivo. J Biol Chem 275: 26411-26415, 2000.

52. Itoh Y, Ito A, Iwata K, Tanzawa K, Mori Y and Nagase H: Plasma membrane-bound tissue inhibitor of metalloproteinases (TIMP)-2 specifically inhibits matrix metalloproteinase 2 (gelatinase A) activated on the cell surface. J Biol Chem 273: 24360-24367, 1998. 